University of South Carolina

Scholar Commons

1990

\title{
Estimation of the Diffusion Coefficient and Solubility for a Gas Diffusing Through a Membrane
}

\author{
Michael C. Kimble \\ Texas A \& M University - College Station \\ Ralph E. White \\ University of South Carolina - Columbia, white@cec.sc.edu \\ Yu-Min Tsou \\ R. Neal Beaver
}

Follow this and additional works at: https://scholarcommons.sc.edu/eche_facpub

Part of the Chemical Engineering Commons

\author{
Publication Info \\ Journal of the Electrochemical Society, 1990, pages 2510-2514. \\ (c) The Electrochemical Society, Inc. 1990. All rights reserved. Except as provided under U.S. copyright law, \\ this work may not be reproduced, resold, distributed, or modified without the express permission of The \\ Electrochemical Society (ECS). The archival version of this work was published in the Journal of the \\ Electrochemical Society. \\ http://www.electrochem.org/ \\ DOI: $10.1149 / 1.2086977$ \\ http://dx.doi.org/10.1149/1.2086977
}

This Article is brought to you by the Chemical Engineering, Department of at Scholar Commons. It has been accepted for inclusion in Faculty Publications by an authorized administrator of Scholar Commons. For more information, please contact digres@mailbox.sc.edu. 


\title{
Estimation of the Diffusion Coefficient and Solubility for a Gas Diffusing Through a Membrane
}

\author{
Michael C. Kimble* and Ralph E. White** \\ Department of Chemical Engineering, Texas A\&M University, College Station, Texas 77843-3122 \\ Yu-Min Tsou and R. Neal Beaver** \\ Dow Chemical USA, Texas Applied Science and Technology Laboratories, Freeport, Texas 77541
}

\begin{abstract}
Analysis of the data obtained by the electrochemical monitoring technique for diffusion of a gas through a membrane is considered. It is shown that combining a numerical method with a nonlinear parameter estimation technique provides a means to determine values for the diffusion coefficient and the solubility of the diffusing gas. It is shown that better accuracy can be obtained for the diffusion coefficient and solubility of this gas by using the method presented and all experimental data rather than only part of the data, as has often been done in the past.
\end{abstract}

The electrochemical monitoring technique developed by Devanathan and Stachurski (1) has been routinely applied to determine diffusion coefficients and solubilities for gases which diffuse through membranes. This technique has been described elsewhere $(2,3)$ but basically consists of first applying a platinum coating to one side of the membrane and then exposing this side of the membrane to the electrolyte and the other side of the membrane to a diffusant gas. A schematic of the overall experimental apparatus is shown in Fig. 1 and a detailed schematic of the permeation test cell is shown in Fig. 2. During the experiment, the gas (e.g., $\mathrm{H}_{2}$ ) diffuses through the membrane and is oxidized electrochemically on the platinum coating. To analyze such a system, a one-dimensional form of Fick's second law of diffusion is used

$$
\frac{\partial c(x, t)}{\partial t}=D \frac{\partial^{2} c(x, t)}{\partial x^{2}}
$$

where the diffusion coefficient, $D$, is assumed to be constant and $c(x, t)$ represents the concentration of the diffusant gas (e.g., $\mathrm{H}_{2}$ ). Initially, an inert gas such as $\mathrm{N}_{2}$ is passed over the membrane which gives

$$
c(x, t)=0.0 \text { for } 0 \leq x \leq L \text { for } t<0
$$

The diffusant gas is assumed to saturate the surface of the membrane which faces the gas chamber upon introduction of the gas to the membrane

$$
c(x, t)=C_{0} \text { at } x=0 \text { for } t \geq 0
$$

At the other side of the membrane, it is assumed that the concentration of $\mathrm{H}_{2}$ is forced to zero by oxidizing all of the $\mathrm{H}_{2}$ gas under mass-transfer limited conditions

$$
c(x, t)=0.0 \text { at } x=L \text { for } t \geq 0
$$

The current as a function of time needed to oxidize the hydrogen gas is given by

$$
i(t)=-\left.n_{\mathrm{e}} \mathbf{F} A D \frac{\partial c(x, t)}{\partial x}\right|_{x=L}
$$

where at steady state the limiting current is

$$
i_{\infty}=\frac{n_{\mathrm{e}} \mathbf{F} A D C_{\mathrm{o}}}{L}
$$

Various analytical methods have been used to approximate the solution of Eq. [1]-[4] in terms of current ratios by using Eq. [5] and [6] as demonstrated by McBreen et al. (4) for the Laplace method

\footnotetext{
* Electrochemical Society Student Member

** Electrochemical Society Active Member.
}

$$
\frac{i(\tau)}{i_{\infty}}=\frac{2}{\sqrt{\pi \tau}} \exp \left(\frac{-1}{4 \tau}\right)
$$

and Fourier's method (4)

$$
\frac{i(\tau)}{i_{\infty}}=1-2 \exp \left(-\pi^{2} \tau\right)
$$

where

$$
\tau=\frac{D t}{L^{2}}
$$

A third analytical solution was presented recently by Yen and Shih (5)

$$
\frac{i(\tau)}{i_{\infty}}=1-\exp (-6 \tau)
$$

Unfortunately, Eq. [7], [8], and [10] are not correct over the entire range of $\tau$ despite being derived from well-known analytical methods. This can be seen easily, for example, by inspection of Eq. [7]. The right-hand side of Eq. [7] goes to zero for large values of $\tau$ instead of going to one, as required. Since the right-hand side of Eq. [7] is the first term only in an infinite series given by (4)

$$
\frac{i(\tau)}{i_{\infty}}=\frac{2}{\pi^{1 / 2}} \frac{1}{\tau^{1 / 2}} \sum_{n=0}^{\infty}(-1)^{n} \exp \left[-\frac{(2 n+1)^{2}}{4 \tau}\right]
$$

one might expect that adding additional terms would improve the accuracy of Eq. [7]. Unfortunately, adding a large (e.g., $10^{6}$ ) number of terms does not improve the solution at all. This is true because $\tau^{1 / 2}$ appears in the denominator for each term, as shown in Eq. [11]. Equation [8] is wrong because the right-hand side does not go to zero for small values of $\tau$, as required. Equation [10] is wrong because, even though it is correct for large and small values of $\tau$, it does not agree with the numerical solution of Eq. [1]-[4] except at one intermediate value of $\tau$, as shown in Fig. 3 .

The numerical solution to Fick's second law, shown in Fig. 3, was calculated by expressing Eq. [1] in a finite difference form (Crank-Nicolson) and solving for the concentration as a function of time at each nodal point subject to the boundary and initial conditions given by Eq. [2]-[4]. The current ratio was then predicted, as a function of time, by using Eq. [5] and [6]. The correct dependence of $i / i_{x}$ on $\tau$ should follow that given by the numerical solution. As can be seen in Fig. 3, the prediction based on the Laplace method (Eq. [7]) deviates from the numerical solution for values of $\tau$ greater than about 0.40 . The prediction based on Fourier's method (Eq. [8]) gives a valid response for $\tau$ 


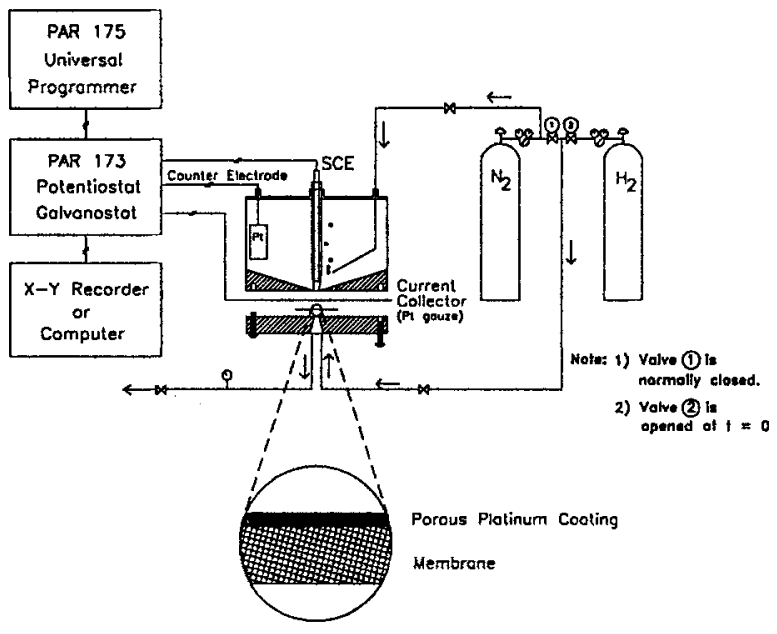

Fig. 1. Schematic of the overall experimental apparatus used for the electrochemical monitoring technique.

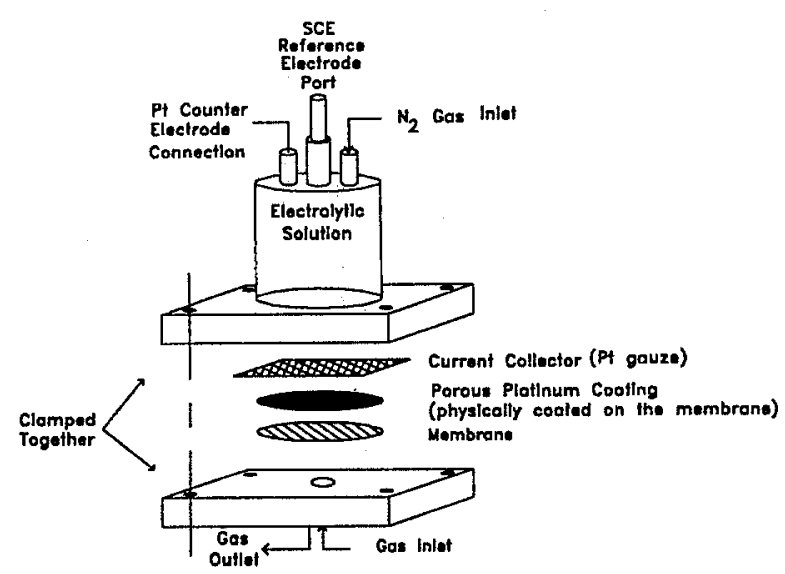

Fig. 2. Schematic of the permeation test cell

greater than about 0.12 , and the prediction according to Eq. [10] deviates from the numerical solution for most of the values of $\tau$. Hence, the numerical method is the only accurate solution to Eq. [1]-[4] over the entire range of $\tau$.

Equations [6] and [9] and one of the Eq. [7], [8], or [10] are often used to determine the diffusion coefficient, $D$, and the solubility, $C_{0}$, of the diffusing gas. The classical method typically consists of setting $i(\tau) / i_{\infty}$ in one of Eq. [7], $[8]$, or [10] to a set fraction (e.g., 0.5) and solving for the dimensionless time, $\tau$. Then the actual time, $t$, to reach this fraction of the limiting current is measured from an experimental current transient. The dimensionless time, $\tau$, and the actual time, $t$, are then used in Eq. [9] with a known thickness, $L$, to calculate $D$. The gas solubility, $C_{0}$, is then calculated from Eq. [6] using the experimentally determined value of $i_{\infty}$. This procedure used with Eq. [10] would lead to significant errors in $D$ and $C_{o}$ if the selected current ratio, $i(\tau) / i_{\infty}$, was not about 0.65 , as indicated in Fig. 3 . This classical procedure is often hard to use because it is difficult to obtain reproducibly flat limiting current curves. Also, one would like to have confidence intervals for $D$ and $C_{o}$ which cannot be obtained when only two values of the experimentally measured current $v s$. time data are used.

The entire data set from an experimentally measured current transient can be used to determine values for $D$ and $C_{0}$ and their confidence intervals by using a numerical solution technique to predict the current, $i$, and the nonlinear parameter estimation procedure to determine $D$ and $C_{0}$ by comparison of the experimental values of $i$ to those predicted by the model (Eq. [1]-[4]). Since the data set consists of $n$ current values and $m$ unknown parameters ( $D$ and $C_{0}$ ), $n$ functions can be defined

$$
f(j)=i_{\mathrm{e}}(j)-\mathrm{i}(j) \quad j=1,2, \ldots, n
$$

where $i_{e}(j)$ represents the $j^{\text {th }}$ experimental value for the current. Here the $n$ current values are obtained at regular intervals from the data set for simplicity.

The theory of least squares fitting can be used to determine $D$ and $C_{0}$. The difference between the experimental values and the predicted values of the current for each data point can be used to determine the two parameters by minimizing the value of $R$ when $R$ is defined as

$$
R=\sum_{j=1}^{n} f(j)^{2}
$$

The IMSL (6) subroutine BCLSF was used in this work to estimate $D$ and $C_{0}$. This routine solves nonlinear least squares problems by using a modified LevenbergMarquardt algorithm. Since $i(j)$ depends on the diffusion coefficient and the solubility, Eq. [1], subject to Eq. [2]-[4], must be solved numerically for each iteration in the estimation of $D$ and $C_{o}$. Fortunately, this does not require much computer time because of the high speed of modern computers.

In addition to estimating the parameters, it is equally important to determine confidence intervals for the parameters. Assuming a normal distribution, the confidence intervals can be approximated by [(7), p. 197]

$$
P_{\mathrm{k}}=\hat{P}_{\mathrm{k}} \pm t_{1-\gamma / 2, \mathrm{df}} \boldsymbol{S}_{\hat{\mathrm{P}}_{\mathrm{k}}} \sqrt{C_{\mathrm{kk}}} \quad k=1, m
$$

where $\hat{P}_{\mathrm{k}}$ is the estimate of the parameter $P_{\mathrm{k}}$ and $t_{1-\gamma / 2, \mathrm{df}}$ is the value of the $t$-distribution at the $(1-\gamma / 2) \times 100 \%$ confidence interval with $n-m$ degrees of freedom (df). A value for the variance, $s_{\mathrm{P}_{\mathrm{k}}}$, can be obtained from

$$
\mathbf{s}_{\hat{\mathrm{P}}_{\mathbf{k}}}{ }^{2}=\frac{\sum_{j=1}^{n} f(j)^{2}}{n-m}
$$

and a value for $C_{\mathrm{kk}}$ can be obtained from the inverse of the approximate Hessian matrix, $\mathbf{N}$, where the elements of the Hessian matrix are given by

$$
\begin{aligned}
H_{\alpha \beta}=\frac{\partial^{2} R}{\partial P_{\alpha} \partial P_{\beta}} & =-2 \sum_{j=1}^{n} f(j) \frac{\partial^{2} i(j)}{\partial P_{\alpha} \partial P_{\beta}} \\
& +2 \sum_{j=1}^{n} \frac{\partial i(j)}{\partial P_{\alpha}} \frac{\partial i(j)}{\partial P_{\beta}} \quad \alpha, \beta=1, m
\end{aligned}
$$

and the elements of the approximate Hessian matrix are

$$
N_{\alpha \beta}=2 \sum_{j=1}^{n} \frac{\partial i(j)}{\partial P_{\alpha}} \frac{\partial i(j)}{\partial P_{\beta}}
$$

The approximate Hessian matrix is a good approximation to the Hessian matrix because as the parameters, $P_{\mathrm{k}}$, approach their final values, the second derivative terms in Eq. [16] tend toward zero. For the two unknown parameters of interest here, $D$ and $C_{o}$, the matrix $\mathbf{N}$ is given by

$$
\mathbf{N}=\left[\begin{array}{cc}
2 \sum_{j=1}^{n} \frac{\partial i(j)}{\partial P_{\mathrm{D}}} \frac{\partial i(j)}{\partial P_{\mathrm{D}}} & 2 \sum_{j=1}^{n} \frac{\partial i(j)}{\partial P_{\mathrm{D}}} \frac{\partial i(j)}{\partial P_{\mathrm{C}_{\mathrm{o}}}} \\
2 \sum_{j=1}^{n} \frac{\partial i(j)}{\partial P_{\mathrm{C}_{o}}} \frac{\partial i(j)}{\partial P_{\mathrm{D}}} & 2 \sum_{j=1}^{n} \frac{\partial i(j)}{\partial P_{\mathrm{C}_{o}}} \frac{\partial i(j)}{\partial P_{\mathrm{C}_{\mathrm{o}}}}
\end{array}\right]
$$

Inverting Eq. [18] and taking the diagonal elements gives the needed values for $C_{\mathrm{kk}}$ in Eq. [14].

\section{Discussion}

To illustrate this procedure for determining estimates and confidence intervals for $D$ and $C_{0}$, the parameters in Table I were used to create a simulated base data set of evenly spaced points of current $v$ s. time. Using simulated data allows the diffusion coefficient and solubility to be set a priori and then calculated by the four methods presented here. The Crank-Nicolson method was used to solve the model Eq. [1]-[4], with $\Delta x=1.0 \times 10^{-4}$ (101 node points) and $\Delta t=7.5 \times 10^{-4}$ (1001 time steps). From this 


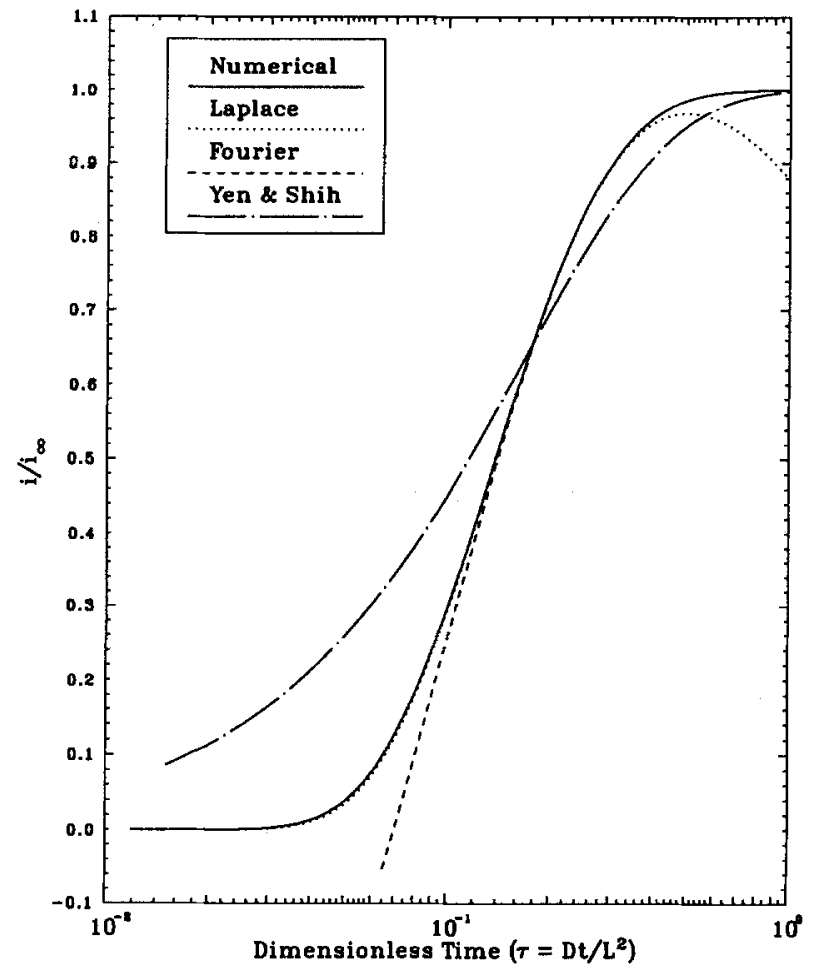

Fig. 3. Comparison of approximote solutions to a numerical solution for the fraction attainment of the steady-state current as a function of the dimensionless time $(\tau)$.

large set of values for $c(x, t)$, values for $i(t)$ were calculated according to Eq. [5] for one thousand evenly spaced points in time over the 30 s time period of the simulated experiment. Since most actual measurements of current transients involve sampling noise, a normal (gaussian) random number generator (subroutine RNNOR of IMSL) was used to alter these currents by $\pm 0.5 \mu \mathrm{A}$ as shown in Fig. 4 . A random deviation of $\pm 0.5 \mu \mathrm{A}$ provides a reasonable amount of induced noise in the current transient resembling a worst

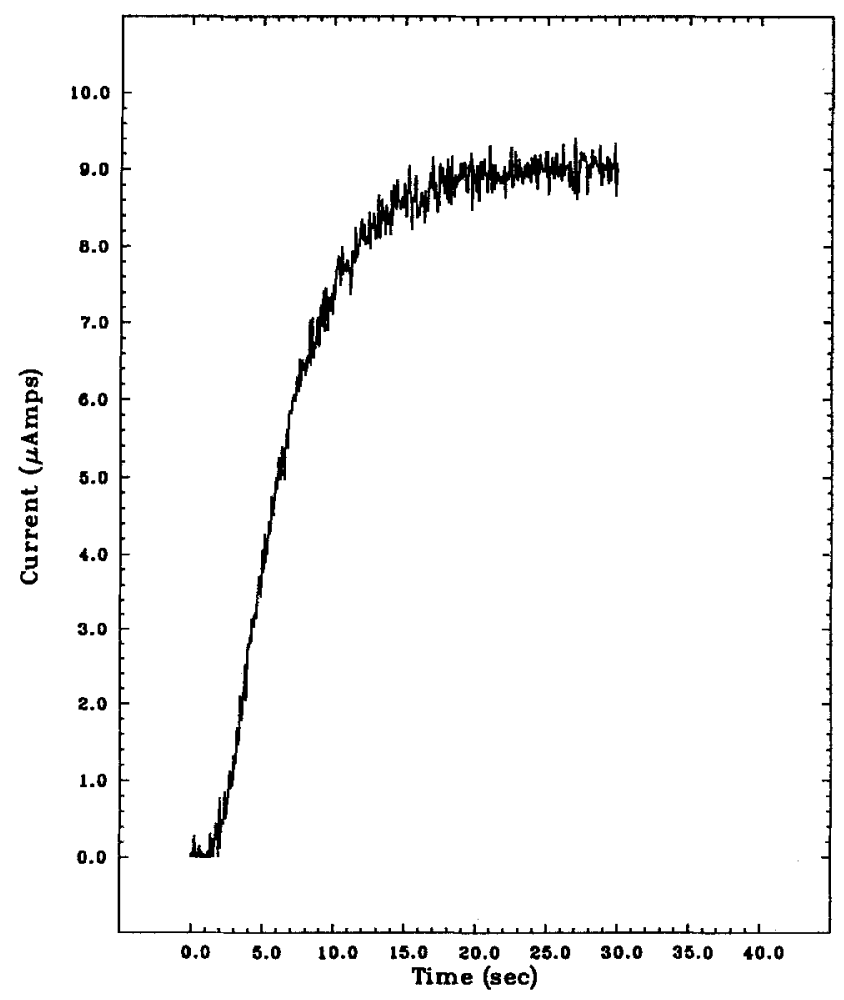

Fig. 4. Computer-generated currents bosed on the numerical solution of Eq. [1]-[4] with randomized induced noise of $\pm 0.5 \mu \mathrm{A}$ (500 evenly spaced points).
Table I. Parameters for the computer generated simulated data ${ }^{\circ}$

$$
\begin{aligned}
& A=0.125 \mathrm{~cm}^{2} \\
& D=2.5 \times 10^{-6} \mathrm{~cm}^{2} / \mathrm{s} \\
& C_{\mathrm{o}}=1.5 \times 10^{-6} \mathrm{~mol} / \mathrm{cm}^{3} \\
& L=0.01 \mathrm{~cm} \\
& n_{\mathrm{e}}=2
\end{aligned}
$$

${ }^{a}$ Selected arbitrarily.

Table II. Calculated parameters and confidence intervals ${ }^{a}$ using

Eq. [19] and [20] for 100 sets of simulated data as o function of the number of data points used in the estimation

\begin{tabular}{ccc}
\hline Data points & $\begin{array}{c}\text { Diffusion coefficient }\left(\times 10^{6}\right) \\
\left(\mathrm{cm}^{2} / \mathrm{s}\right)\end{array}$ & $\begin{array}{c}\text { Solubility }\left(\times 10^{6}\right) \\
\mathrm{mol} / \mathrm{cm}^{3}\end{array}$ \\
\hline 50 & $2.4967 \pm 0.006477$ & $1.5036 \pm 0.004893$ \\
100 & $2.5012 \pm 0.004013$ & $1.5000 \pm 0.002991$ \\
150 & $2.5022 \pm 0.003117$ & $1.4986 \pm 0.002395$ \\
200 & $2.5006 \pm 0.002768$ & $1.4993 \pm 0.001847$ \\
250 & $2.4994 \pm 0.002394$ & $1.5010 \pm 0.001717$ \\
300 & $2.5000 \pm 0.002214$ & $1.4992 \pm 0.001577$ \\
350 & $2.5007 \pm 0.001845$ & $1.4998 \pm 0.001396$ \\
400 & $2.5010 \pm 0.001618$ & $1.4991 \pm 0.001308$ \\
450 & $2.4998 \pm 0.001588$ & $1.5001 \pm 0.001222$ \\
500 & $2.4992 \pm 0.001473$ & $1.5005 \pm 0.001070$ \\
\hline
\end{tabular}

a5\% confidence interval.

case data set. Since random deviates were added to the base data set, a Monte Carlo simulation [(8) p. 46] is needed to illustrate adequately the numerical method and parameter estimation technique.

The Monte Carlo simulation consists of numerous repetitions of generating and analyzing simulated data sets. That is, numerous simulated data sets are created by first calculating a (smooth) current transient as described above and, second, by randomly altering this current transient numerous times ( $r$ repetitions) resulting in many simulated data sets similar to Fig. 4. Once these simulated data sets are created, the numerical method, Eq. [1]-[4], and parameter estimation technique is used to analyze individually each data set. Each repetition is used to estimate a diffusion coefficient, $D_{1}$, and a solubility parameter, $C_{01}$.

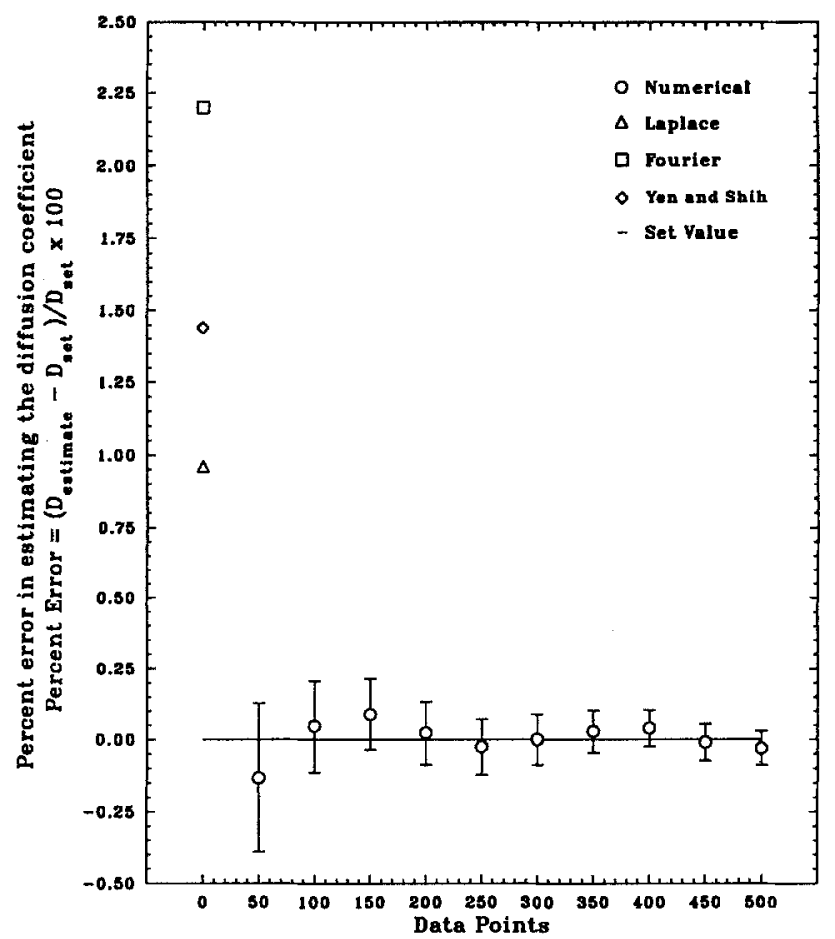

Fig. 5. Variation of the percent error in estimating the diffusion coefficient with the number of evenly spaced data points used in the parameter estimation within $95 \%$ confidence. 


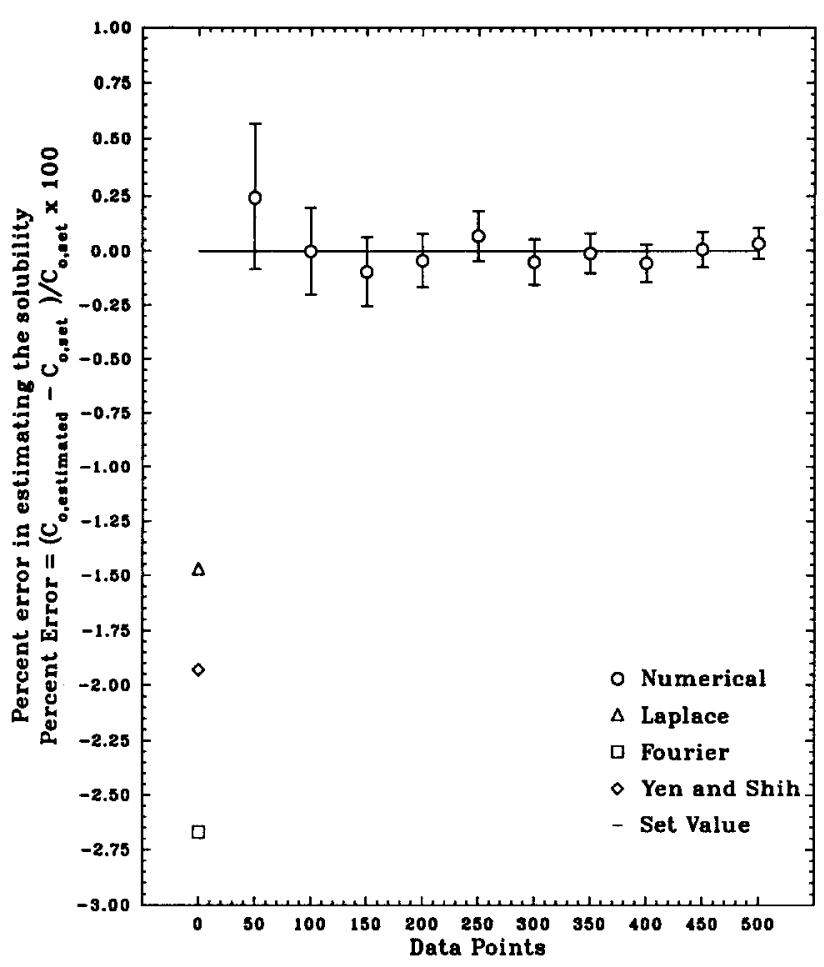

Fig. 6. Variation of the percent error in estimating the solubility with the number of evenly spaced data points used in the parameter estimation within $95 \%$ confidence.

For the Monte Carlo simulation, the parameters and their confidence intervals are determined from all $r$ repetitions of the simulation $[(9) \mathrm{p} .226]$

$$
P_{\mathrm{k}}=\bar{P}_{\mathrm{k}} \pm t_{1-\gamma / 2, \mathrm{df}} \frac{s_{\mathrm{k}}}{\sqrt{r}} \quad k=1, m
$$

where $\bar{P}_{1}$ and $\bar{P}_{2}$ are the average values for $D_{1}$ and $C_{o_{1}}$, respectively, over all $r$ repetitions, $d f=r-1$, and $s_{\mathrm{k}}$ is the sample standard deviation of parameter $k$ given by

$$
s_{\mathrm{k}}=\left[\sum_{l=1}^{r} \frac{\left(P_{\mathrm{k}_{1}}-\bar{P}_{\mathrm{k}}\right)^{2}}{r-1}\right]^{1 / 2} \quad k=1, m
$$

Note that Eq. [19] and [20] and the Monte Carlo simulation are only needed to analyze the computer generated data (Fig. 4). If real data is being analyzed, Eq. [14] is used to estimate the confidence intervals.

One hundred data sets like the one shown in Fig. 4 were generated with 500 data points and analyzed by the numerical method and parameter estimation technique. The resulting 100 values for the diffusion coefficient and solubility were used in Eq. [19] and [20] to obtain the parameter estimates and their confidence intervals. The results for this case together with other cases with fewer data points but with 100 repetitions for each are shown in Table II. Figures 5 and 6 show the percent errors for the diffusion coefficient and solubility, respectively. These results show that the confidence interval for the parameter estimates become smaller as more simulated data points are used. This

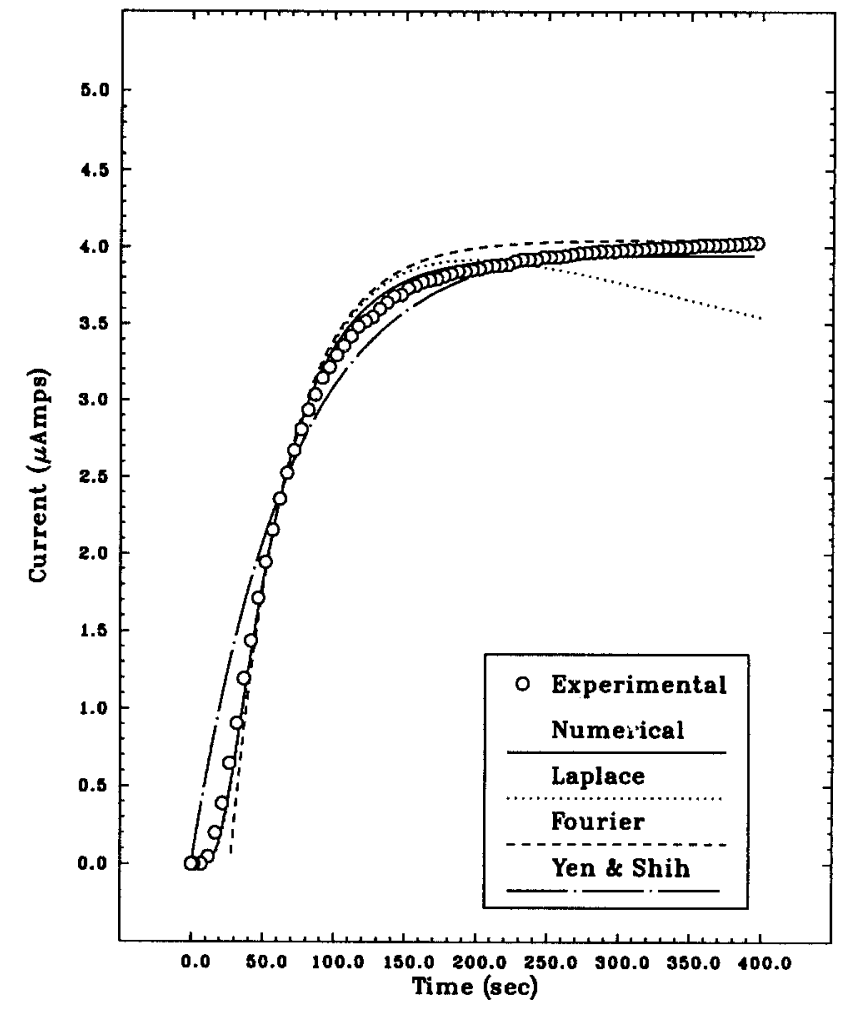

Fig. 7. Experimentally measured and predicted values of current as a function of time for hydrogen gas diffusing through a proprietary ionexchange membrane ( 82 evenly spaced in time data points).

observation is expected since as the number of data points increases, the parameter estimates will approach their true values and the confidence intervals will tend toward zero.

Also shown in Fig. 5 and 6 are percent errors in the values for $D$ and $C_{0}$ obtained by applying the three approximate solutions, Eq. [7], [8], and [10], with the classical method described above to the simulated data in Fig. 4. Setting $i(\tau) / i_{\infty}$ to 0.5 in Eq. [7] and [8] and solving for $\tau$ gives $\tau_{\text {Laplace }}=0.1388$ and $\tau_{\text {Fourier }}=0.1405$. Equation [10] provides the correct value for $\tau$ at $i(\tau) / i_{\infty}$ equal to 0.65 as shown in Fig. 3. Using this value in Eq. [10] gives $\tau_{\text {Yen and Shih }}=0.1750$. The limiting current, as approximated from Fig. 4 by using a ruler is $9 \mu \mathrm{A}$. The time, $t$, to reach one-half of the limiting current is $5.5 \mathrm{~s}$ and to obtain 0.65 of the limiting current is $6.9 \mathrm{~s}$. The diffusion coefficients for the Laplace and Fourier equations are obtained from Eq. [9] using $t=5.5 \mathrm{~s}$ and the respective values for $\tau$ above. Similarly, the diffusion coefficient for the Yen and Shih equation is calculated by using $t=6.9 \mathrm{~s}$ and the above corresponding value of $\tau$. The solubility can then be calculated for each approximate method by using the calculated diffusion coefficients and approximated limiting current in Eq. [6]. Table III presents a comparison of these results to the numerical method. It is probably not necessary to analyze graphically each of the 100 data sets since the only difference between each data set is the amount of randomly induced noise. As shown in Table III and Fig. 5 and 6, the numerical method gives a more accurate estimate of the diffusion coefficient and solubility than the approximate methods. It should be

\begin{tabular}{|c|c|c|c|c|}
\hline Method & $\begin{array}{l}D\left(\times 10^{6}\right) \\
\left(\mathrm{cm}^{2} / \mathrm{s}\right)\end{array}$ & $\begin{array}{l}\% \text { Error }^{\mathrm{a}} \\
\text { in } D\end{array}$ & $\begin{array}{c}\mathrm{C}_{o}\left(\times 10^{6}\right) \\
\left(\mathrm{mol} / \mathrm{cm}^{3}\right)\end{array}$ & $\begin{array}{l}\% \text { Error }^{b} \\
\text { in } C_{0}\end{array}$ \\
\hline $\begin{array}{l}\text { Numerical }^{\mathrm{c}} \\
\text { Laplace } \\
\text { Fourier } \\
\text { Yen }\end{array}$ & $\begin{array}{c}2.4967 \pm 0.006477 \\
2.524 \\
2.555 \\
2.536\end{array}$ & $\begin{array}{c}-0.132 \\
0.960 \\
2.20 \\
1.44\end{array}$ & $\begin{array}{c}1.5036 \pm 0.004893 \\
1.478 \\
1.460 \\
1.471\end{array}$ & $\begin{array}{l}0.240 \\
-1.47 \\
-2.67 \\
-1.93\end{array}$ \\
\hline
\end{tabular}

Table III. Comparison of values obtained for $D$ and $C_{o}$ by different methods for simulated data

a Relative to the set value of $2.5 \times 10^{-6} \mathrm{~cm}^{2} / \mathrm{s}$.

b Relative to the set value of $1.5 \times 10^{-6} \mathrm{~mol} / \mathrm{cm}^{3}$.

c Obtained with 50 evenly spaced data points. 
Table IV. Comparison of values obtained for $D$ and $C_{o}$ by different methods for experimental dato

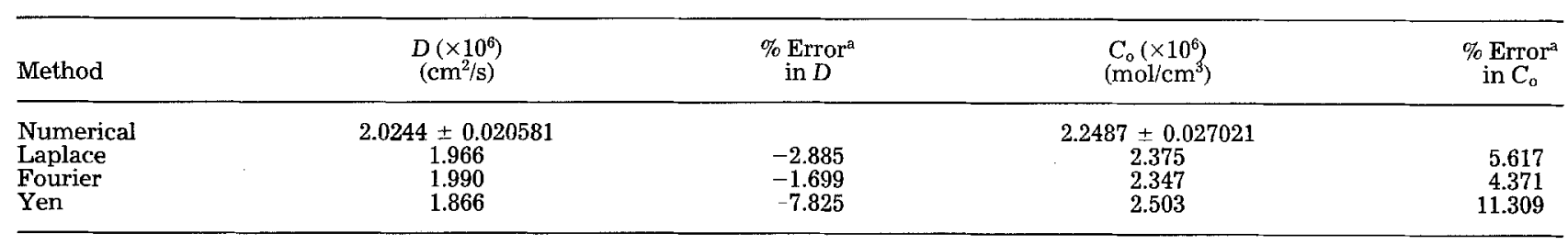

a Relative to the numerical method.

mentioned that more than one data point from the current transient could be used to perform the estimation for the approximate methods. However, this would lessen the appeal of the approximate methods and may be more diffcult than using the numerical method.

To further illustrate the numerical method technique for estimating diffusion coefficients and solubilities, actual experimental data was analyzed by the four methods described above. The permeation rate of hydrogen gas through a proprietary membrane was measured by the electrochemical monitoring technique as described earlier. The temperature of the electrolysis cell was $25^{\circ} \mathrm{C}$ and consisted of a $0.5 \mathrm{M} \mathrm{Na}_{2} \mathrm{SO}_{4}$ electrolyte and a 11.13 mil thick membrane with an exposed geometric area of $0.125 \mathrm{~cm}^{2}$. From the resulting current transient, 82 evenly spaced in time data points were obtained as shown in Fig. 7. Although the experimental current transient in Fig. 7 appears to be smooth, these values were obtained from a measured current transient similar to Fig. 4. Also shown in Fig. 7 are the predicted current transients obtained from the numerical method and the three approximate methods. The model Eq. [1]-[4], were solved by the CrankNicolson method with $\Delta x=2.783 \times 10^{-4} \mathrm{~cm}$ (101 node points) and $\Delta t=2.678 \times 10^{-2} \mathrm{~s}(14,936$ time steps). Since real experimental data points were used rather than simulated data, the confidence intervals were calculated by using Eq. [14]. The three approximate methods were also applied to the experimental data. Using Fig. 7, the limiting current is approximately $4.047 \mu \mathrm{A}$. One-half of this limiting current value corresponds to a time of $54.69 \mathrm{~s}$ and 0.65 of the limiting current corresponds to $72.66 \mathrm{~s}$. The results for the hydrogen gas diffusion coefficient and solubility as obtained by the four methods are shown in Table IV. These values for the diffusion coefficient and solubility were then used in their respective equations (Eq. [5]-[10]) to predict the current as a function of time as shown in Fig. 7 .

\section{Summary}

A numerical solution to Fick's second law of diffusion can be used with transient current data to obtain estimates and confidence intervals for the diffusion coefficient and solubility of a gas diffusing through a membrane. Higher accuracy is obtained in estimating the diffusion coefficient and solubility by using a numerical method rather than approximate methods.

There are several advantages in using a numerical method with parameter estimation as presented here. First, the estimated parameters depend on all experimental data, not just on two points as they do for the approximate methods. Hence, there is less chance of an error occurring in the estimation. Also, a confidence interval can be constructed for the parameter estimate with only one set of experimental data. Second, the approximate solutions depend on the limiting current value which is sometimes difficult to measure (as in Fig. 4) and may vary on subsequent experimental runs. This can significantly alter the parameter estimates. Third, the use of high speed digital computers provides a simple, rapid method to determine parameter estimates and their confidence intervals. Coupling data acquisition hardware and software with such computers would make the approach presented here even more appealing.

\section{Acknowledgment}

The authors are grateful for the support of this project by Dow Chemical USA and the State of Texas through its
Texas Advanced Technology and Research Program. Also, the authors would like to thank Dr. A. T. Watson of the Department of Chemical Engineering, Texas A\&M University, for his helpful suggestions throughout this work.

Manuscript submitted Oct. 12, 1989; revised manuscript received ca. Feb. 26, 1990.

Texas A\&M University assisted in meeting the publication costs of this article.

\section{LIST OF SYMBOLS}

A cross-sectional area of membrane and electrode, $\mathrm{cm}^{2}$

c concentration of gas in the membrane, $\mathrm{mol} / \mathrm{cm}^{3}$

$C_{\mathrm{kk}}$ diagonal matrix element of $\mathbf{N}^{-1}$

$\mathrm{C}_{0}$ diffusing gas solubility in the membrane, $\mathrm{mol} / \mathrm{cm}^{3}$

$\mathrm{C}_{\mathrm{O}_{\mathrm{l}}}$ diffusing gas solubility of repetition $l, \mathrm{~mol} / \mathrm{cm}^{3}$

$D$ diffusion coefficient of the diffusing gas in the membrane, $\mathrm{cm}^{2} / \mathrm{s}$

$D_{1} \quad$ diffusion coefficient of repetition $l, \mathrm{~cm}^{2} / \mathrm{s}$

F Faraday constant, 96,487 C/mol

$f(j)$ residual current (experimental-predicted) at time $j, \mathrm{~A}$

H Hessian matrix

$i_{\mathrm{e}}(j) \quad$ experimental current measured at time $j, \mathrm{~A}$

$i(j)$ predicted current measured at time $j, \mathrm{~A}$

$i(t) \quad$ current at time $t, \mathrm{~A}$

$i_{\infty} \quad$ limiting current, $\mathrm{A}$

$L \quad$ membrane thickness, $\mathrm{cm}$

$m \quad$ number of parameters $\left(m=2, D\right.$, and $\left.C_{0}\right)$

$n$ number of experimental observations (data points)

$\begin{array}{ll}n_{\mathrm{e}} & \text { moles of electrons } \\ \mathbf{N} & \text { approximate Hessian matrix }\end{array}$

$\underline{P}_{\mathrm{p}} \quad$ parameter $k$

$\bar{P}_{\mathrm{P}}^{\mathrm{k}} \quad$ arithmetric mean of parameter $k$ over $r$ repetitions

$\hat{P}_{\mathrm{k}} \quad$ estimate of parameter $k$

$r$ number of repetitions

$R \quad$ residual sum of squares of the error, $\mathrm{A}^{2}$

$s_{\mathrm{k}} \quad$ sample standard deviation of parameter $k$

$S_{\hat{P}_{\mathrm{k}}} \quad$ approximate value of the variance of $\hat{\boldsymbol{P}}_{\mathrm{k}}$

$t$ time, $\mathrm{s}$

$t_{1-\gamma / 2, \text { df }} t$-distribution at $(1-\gamma / 2) \times 100 \%$ confidence interval with $d f(=n-m)$ degrees of freedom for Eq. [13] and $d f(=r-1)$ for Eq. [18]

$\tau \quad$ dimensionless time, $D t / L^{2}$

$x \quad$ spatial coordinate, $\mathrm{cm}$

$\gamma \quad$ parameter for the $t$-distribution

\section{REFERENCES}

1. M. A. V. Devanathan and Z. Stachurski, Proc. R. Soc., Edinburgh, Sect. A, 270, 90 (1962).

2. R. S. Yeo and J. McBreen, This Journal, 126, 1682 (1979).

3. Z. Ogumi, Z. Takehara, and S. Yoshizawa, ibid., 131, 769 (1984).

4. J. McBreen, L. Nanis, and W. Beck, ibid., 113, 1218 (1966).

5. S. K. Yen and H. C. Shih, ibid., 135, 1169 (1988).

6. Subroutine BCLSF of IMSL library, in "Problem-Solving Software System for Mathematical and Statistical FORTRAN Programming," User's Manual Version 1.0, IMSL, Inc., Houston, TX 77042 (1987).

7. D. M. Himmelblau, "Process Analysis by Statistical Methods," John Wiley \& Sons, Inc., New York (1970).

8. Y. Bard, "Nonlinear Parameter Estimation," Academic Press, Inc., New York (1974).

9. J. S. Milton and J. C. Arnold, "Probability and Statistic in the Engineering and Computing Sciences," McGraw Hill, Inc., New York (1986). 